\title{
Avaliação da estabilidade térmica de sais quaternários de amônio para uso em argilas organofílicas nacionais - Parte I
}

\section{(Evaluation of thermal stability of quaternary ammonium salts for use in national organoclays - Part I)}

\author{
R. Barbosa', D. D. Souza ${ }^{2}$, E. M. Araújo' ${ }^{2}$ T. J. A. Mélo \\ ${ }^{1}$ Universidade Federal do Piaui, Centro de Tecnologia,Teresina, PI, 64.049-550 \\ ${ }^{2}$ Departamento de Engenharia de Materiais da UFCG, Campina Grande, PB, 58429-900 \\ rrenatabarbosa@yahoo.com,dayannediniz@hotmail.com,karine.nobrega@yahoo.com.br, \\ edcleide@dema.ufcg.edu.br,tomas@dema.ufcg.edu.b
}

\begin{abstract}
Resumo
A grande propagação da nanotecnologia nos tempos atuais requer estudos cada vez mais específicos para entender a evolução desses materiais. A obtenção de boas propriedades em nanocompósitos polímero/argila faz com que estudos mais aprofundados sejam realizados sobre a estabilidade térmica dos surfactantes nas temperaturas de processamento destes materiais. Neste trabalho, a estabilidade térmica de quatro sais quaternários de amônio, tais como brometo de hexadeciltrimetil amônio, cloreto de alquildimetilbenzil amônio, cloreto de estearildimetil amônio e cloreto de hexadeciltrimetil amônio foi avaliada, visando sua posterior intercalação em argilas bentoníticas nacionais, com o propósito de torná-las organofílicas para uso em nanocompósitos poliméricos. Os sais foram caracterizados termicamente por calorimetria exploratória diferencial e termogravimetria sob $\mathrm{Ar}$ e $\mathrm{N}_{2}$. Os resultados indicaram que os sais à base do ânion cloreto se degradaram em temperaturas similares e o sal à base do ânion brometo se degradou em temperatura superior. Além disso, este estudo indicou que o sal cloreto de estearildimetil amônio provavelmente terá sucesso na utilização como surfactante para o uso em nanocompósitos.
\end{abstract}

Palavras-chave: nanocompósitos, argilas organofílicas, sais quaternários de amônio, estabilidade térmica.

\begin{abstract}
The large propagation of nanotechnology in modern times requires more studies to meet their specific development. For obtaining good properties of polymer/clay nanocomposites more comprehensive studies on the thermal stability of surfactants in the processing temperatures of these materials are necessary. In this work, the thermal stability of four quaternary ammonium salts such as hexadecyltrimethyl ammonium bromide, alkyldimethylbenzyl ammonium chloride, esthearildimethyl ammonium chloride and hexadecyltrimethyl ammonium chloride was evaluated for their intercalation into national bentonite clay, with the aim to enable organophilic for using in polymer nanocomposites. The salts were thermally characterized by differential scanning calorimetry and thermogravimetryunder Ar and $N_{2}$. The results indicate that the salts based on chloride ion degraded at similar temperatures and the based on the bromide anion degraded at higher temperature. Besides, these studies indicated that the esthearildimethyl ammonium chloride salt may be successful in obtaining as surfactant for the use in nanocomposite.
\end{abstract}

Keywords: nanocomposites, organoclays, quaternary ammonium salts, thermal stability.

\section{INTRODUÇÃO}

Os avanços tecnológicos na área de nanotecnologia incentivam governos de vários países a investir intensamente em pesquisas que possam trazer melhoras significativas nas propriedades dos diferentes materiais e inovações em síntese e controle desses materiais em escala nanométrica [1]. No contexto de nanotecnologia os nanocompósitos poliméricos tem conseguido atenção por parte da população acadêmica e pesquisas nesta área estão sendo cada vez mais difundidas, especialmente aos nanocompósitos poliméricos desenvolvidos com silicatos em camada, que representam uma alternativa aos compósitos desenvolvidos com cargas convencionais. A adição de níveis mínimos $(<10 \%)$ de argilas organofílicas melhora as propriedades mecânicas, térmicas, de barreira e estabilidade dimensional dos nanocompósitos [2-4]. Para que seja possível a obtenção desses materiais híbridos orgânico-inorgânico se faz necessária a modificação superficial dessas cargas, tornandoas compatíveis quimicamente com a matriz polimérica. Tais argilas recebem o nome de argilas organofílicas. Geralmente a modificação das argilas para torná-las organofílicas ocorre através de reações de troca catiônica com sais quaternários de amônio. Os cátions orgânicos do sal substituem os cátions de sódio da bentonita, passando-a de hidrofílica para organofílica [5], como mostra a Fig. 1. 


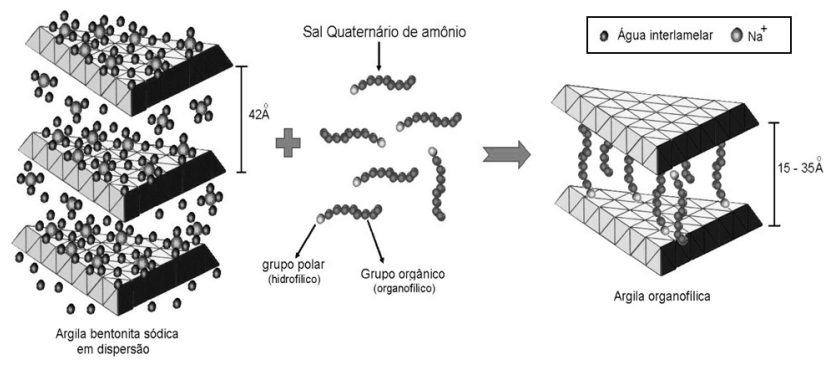

Figura 1: Esquema da introdução do sal orgânico e da substituição dos cátions trocáveis na argila [6].

[Figure 1: Scheme of the introduction of organic salt and replacement of exchangeable cations in clay [6].]

A presença de cátions quaternários de cadeias alquílicas com até 10 átomos de carbono promove a contração da reflexão basal para o valor de 13,6 ̊̊ e a partir de 12 átomos a reflexão basal aumenta para o valor de 17,6 das argilas. As cadeias alquílicas do cátion quaternário de amônio estariam estendidas ao longo das faces lamelares das camadas $2: 1$ e os planos das cadeias zigzag de carbonos estariam paralelos aos planos das faces de oxigênio da camada 2:1 [7]. Os cátions quaternários de cadeia longa, como $\mathrm{C}_{16}$ ou $\mathrm{C}_{18}$, permitem a formação de grandes galerias com maiores diâmetros entre as camadas 2:1 com propriedades hidrofóbicas, ou seja, com aumento do comprimento, isto é, do número de carbonos da molécula linear alquílica é possível conseguir que o cátion quaternário de amônio fique perpendicular ao plano das camadas 2:1 entre as quais está intercalado, provocando com isso um maior inchamento da distância interplanar das argilas [8]. A composição química e as dimensões dos cátions determinam, portanto, a distância interplanar basal da argila organofílica, bem como controlam um conjunto amplo de propriedades, especialmente adsortivas e coloidais, que levam ao seu grande uso industrial atual. Esses cátions diminuem a tensão superficial das bentonitas e melhoram seu caráter de molhamento pela matriz polimérica. Além disso, os cátions alquilamônio e alquilfosfônio podem ter grupos funcionais e reagir com a matriz polimérica ou, em alguns casos, iniciar a polimerização de monômeros melhorando a resistência da interface entre as partículas de argila e a matriz polimérica [9]. Apesar do sucesso relativo da intercalação/esfoliação de argilas por polímeros fundidos, este método ainda apresenta algumas dificuldades práticas que impedem que ele seja utilizado em escala industrial. Além da necessidade da escolha adequada do trio polímero/argila/surfactante, de modo que se obtenha a compatibilidade necessária para uma boa dispersão, é preciso estar atento à estabilidade térmica do surfactante nas temperaturas de processamento dos nanocompósitos. Estudos de degradação verificaram que a decomposição de alguns sais pode começar a ser significativa em temperatura da ordem de $180^{\circ} \mathrm{C}[10]$. Como a maioria dos termoplásticos ao qual a argila organofílica deverá ser incorporada é processada, no mínimo, por volta dessa temperatura, à estabilidade térmica do sal presente na argila deve sempre ser considerada. Certos sais são mais estáveis do que outros, sendo necessário o estudo de cada caso.

Este trabalho realizou um estudo sistemático com quatro sais quaternários de amônio, a fim de estudar a estabilidade térmica destes sais para possíveis intercalações em argilas bentonitícas nacionais, almejando o uso destas argilas em sistemas nanocompósitos poliméricos. Os sais quaternários de amônio foram caracterizados termicamente por calorimetria exploratória diferencial (DSC) e termogravimetria (TG) em duas taxas de aquecimento $10,0^{\circ} \mathrm{C} / \mathrm{min}$ e $12,5^{\circ} \mathrm{C} / \mathrm{min}$ em atmosferas de $\operatorname{Ar}$ e $\mathrm{N}_{2}$.

\section{MATERIAIS E MÉTODOS}

\section{Materiais}

Os sais quaternários de amônio utilizados foram o brometo de hexadecil trimetil amônio de nome comercial Cetremide ${ }^{\circledR}$, fornecido no estado sólido e fabricado pela Vetec S. Paulo, SP. O Dodigen $1611^{\circledR}$ (cloreto de alquil dimetil benzil amônio), fornecido no estado líquido com quantidade de matéria ativa presente de $50 \%$. O Praepagen $\mathrm{WB}^{\circledR}$ (cloreto de estearil dimetil amônio), fornecido no estado de gel com quantidade de matéria ativa constituída de aproximadamente $75 \%$. E o Genamim CTAC-50ET ${ }^{\circledR}$ (cloreto de hexadecil trimetil amônio), fornecido no estado de gel com quantidade de matéria ativa constituída de aproximadamente $50 \%$. Os três últimos sais foram fabricados pela Clariant, Recife, PE. A Fig. 2 mostra as estruturas moleculares dos sais usados.

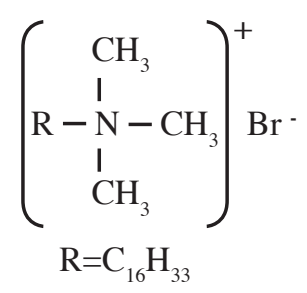

a)

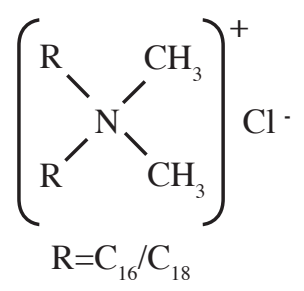

c)

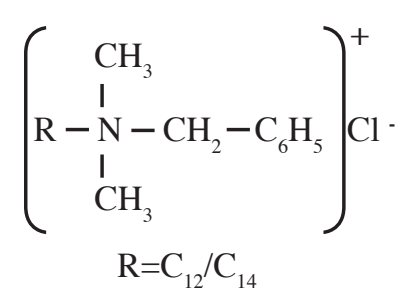

b)

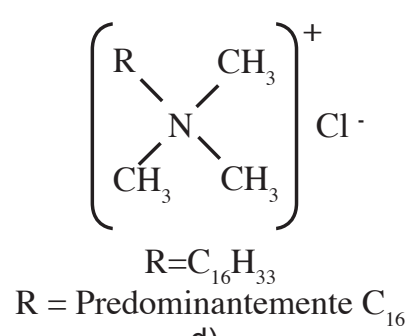

d)
Figura 2: Estruturas moleculares dos sais quaternários de amônio: a) Cetremide ${ }^{\circledR}$ b) Dodigen ${ }^{\circledR}$ c) Praepagen ${ }^{\circledR}$ e d) Genamin ${ }^{\circledR}$.

[Figure 2: Molecular structures of the quaternary ammonium salts: a) Cetremide ${ }^{\circledR}$ b) Dodigen ${ }^{\circledR}$ c) Praepagen ${ }^{\circledR}$ e d) Genamin $^{\circledR}$.] 


\section{Métodos}

Calorimetria Exploratória Diferencial: a verificação da estabilidade térmica dos sais quaternários de amônio foi conduzida por calorimetria exploratória diferencial (DSC50 Shimadzu), a uma taxa de aquecimento de $10{ }^{\circ} \mathrm{C} / \mathrm{min}$ e vazão de nitrogênio da temperatura ambiente até $500{ }^{\circ} \mathrm{C}$. O porta-amostra utilizado foi de alumínio e a massa de amostra de $\sim 10 \mathrm{mg}$. Termogravimetria: as análises foram conduzidas em aparelho TGA-50 Shimadzu. Empregou-se $10 \mathrm{mg}$ de amostra e taxas de aquecimento de $10^{\circ} \mathrm{C} / \mathrm{min}$ e $12,5^{\circ} \mathrm{C} / \mathrm{min}$. As amostras foram aquecidas da temperatura ambiente até $900{ }^{\circ} \mathrm{C}$ sob $\mathrm{Ar}$ e $\mathrm{N}_{2}$ em porta amostras de alumina.

\section{RESULTADOS E DISCUSSÃO}

\section{Calorimetria exploratória diferencial}

As curvas DSC dos sais quaternários de amônio Praepagen ${ }^{\circledR}$ (PRA), Genamin ${ }^{\circledR}$ (GEN), Dodigen ${ }^{\circledR}$ (DOD) e Cetremide ${ }^{\circledR}$ (CET) estão apresentadas na Fig. 3. Para o sal PRA observa-se possivelmente vaporizações primárias e secundárias a $70{ }^{\circ} \mathrm{C}$ e a $112{ }^{\circ} \mathrm{C}$, respectivamente. Essas vaporizações são decorrentes de água e de algum solvente existente em sua formulação, pois em sua composição são encontrados $75 \%$ de matéria ativa e $25 \%$ de solventes. São observados também dois picos de decomposição em 210 ${ }^{\circ} \mathrm{C}$ provavelmente da perda de estrutura do $\mathrm{C}_{16}$ e a $325^{\circ} \mathrm{C}$ referente possivelmente à cadeia de carbono $\mathrm{C}_{18}$, existente na estrutura do sal quaternário de amônio. A curva DSC para o sal GEN apresenta volatilizações primárias e secundárias a $76{ }^{\circ} \mathrm{C}$ e a $100{ }^{\circ} \mathrm{C}$, respectivamente. Aparentemente, o pico de decomposição da estrutura da matéria ativa ocorre a $260{ }^{\circ} \mathrm{C}$. Na formulação do sal GEN é encontrado quantidade de matéria ativa constituída de aproximadamente $50 \%$ e consequentemente $50 \%$ de solventes. Para o sal DOD observa-se volatilização a 75 ${ }^{\circ} \mathrm{C}$ e uma pequena intensidade em $115^{\circ} \mathrm{C}$, provavelmente de uma volatilização secundária. A decomposição do sal ocorre na temperatura do pico de $200^{\circ} \mathrm{C}$. Em sua formulação é encontrada quantidade de matéria ativa constituída de aproximadamente 50\%, semelhante ao sal GEN. O CET é o sal quaternário de amônio que foi fornecido no estado sólido e observa-se que a temperatura de fusão ocorre a $115{ }^{\circ} \mathrm{C}$ e que a sua decomposição ocorre na temperatura do pico de $270{ }^{\circ} \mathrm{C}$, com uma banda endotérmica a $305^{\circ} \mathrm{C}$. Analisados por DSC verificam-se que os sais quaternários de amônio GEN e CET são mais estáveis termicamente e com valores próximos de temperaturas de decomposição. Isto se deve provavelmente, à semelhança de estruturas moleculares e ao mesmo número de carbonos existentes em suas composições [10-12]. Visando um posterior uso destas argilas organofílicas em nanocompósitos onde a temperatura de processamento é de aproximadamente 200 ${ }^{\circ} \mathrm{C}$, aparentemente todos os sais com exceção do DOD poderiam ser usados para a organofilização da argila e consequientemente nos sistemas de nanocompósitos.

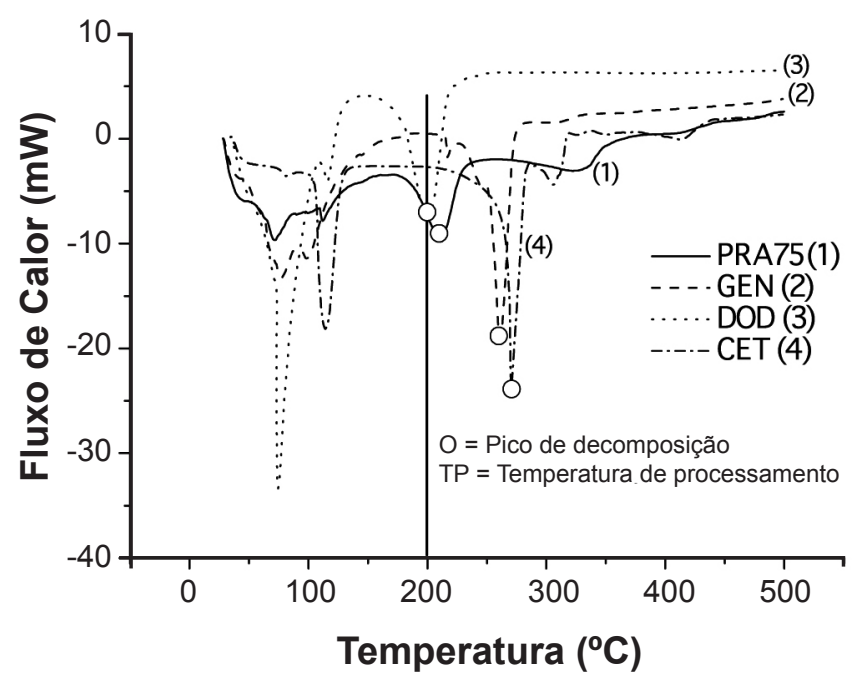

Figura 3: Curvas DSC dos sais quaternários de amônio. [Figure 3: DSC curves of the quaternary ammonium salts.]

\section{Termogravimetria}

A Fig. 4 apresenta as curvas de termogravimetria dos sais quaternários de amônio nas atmosferas de ar e em nitrogênio sob duas taxas de aquecimento, $10^{\circ} \mathrm{C} / \mathrm{min}$ e 12,5 ${ }^{\circ} \mathrm{C} / \mathrm{min}$. As estruturas dos sais quaternários também estão na mesma figura. Observa-se no geral que o comportamento é semelhante, independente das taxas de aquecimento e dos gases. Verifica-se que os sais à base do anion cloreto começam a se degradar em temperaturas similares, enquanto o sal à base do ânion brometo se degrada em temperatura superior. Estes resultados estão de acordo com os reportados anteriormente por calorimetria exploratória diferencial. O tipo de ânion pode influenciar a estabilidade térmica do sal quaternário de amônio e os sais constituídos do ânion $\mathrm{Cl}^{-}$são freqüentemente menos estáveis do que outros tipos de ânions. $\mathrm{O} \mathrm{Cl}^{-}$é um forte nucleofílico e esses ânions se decompõem termicamente via substituição nucleofílica [13], o que foi comprovado neste estudo. O sal DOD possui tamanhos de cadeias carbônicas inferiores aos sais PRA, GEN e CET, resultando provavelmente em sua menor estabilidade. Outra explicação está no fato que o sal DOD possui em sua estrutura a presença do grupo benzil que é bastante eletrofílico facilitando o ataque ao ânion cloreto. Os demais sais quaternários de amônio possuem tamanhos de cadeias carbônicas de $\mathrm{C}_{16}$, sendo o PRA constituído de uma mistura de $\mathrm{C}_{16}$ e $\mathrm{C}_{18}$. São observadas para esse sal duas etapas de decomposição, uma referente provavelmente ao $\mathrm{C}_{16}$ e a outra referente ao $\mathrm{C}_{18}$ que o torna mais estável do que o sal CET. É visto também para o sal PRA que em temperaturas acima de $300{ }^{\circ} \mathrm{C}$ a estabilidade térmica é superior aos demais sais. Os grupos benzil e metil presentes na constituição dos sais quaternários de amônio são mais susceptíveis ao ataque nucleofílico favorecendo a perda de estabilidade térmica e que o caminho predominante na decomposição dos sais puros é através do ataque do íon cloreto no grupo metil formando respectivamente amina livre e clorometano e/ou 

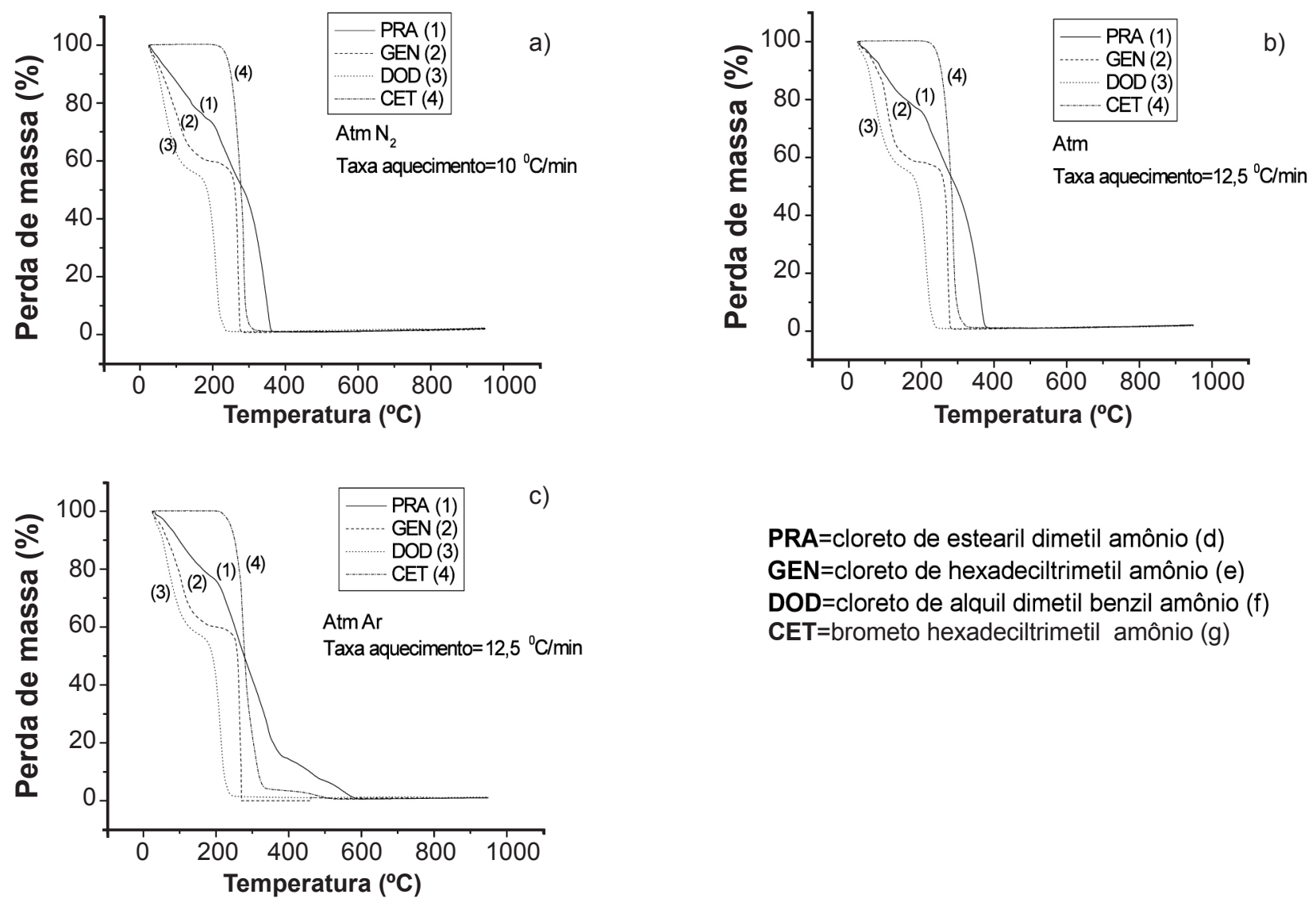

PRA=cloreto de estearil dimetil amônio (d) GEN=cloreto de hexadeciltrimetil amônio (e) $\mathrm{DOD}=$ cloreto de alquil dimetil benzil amônio (f) CET=brometo hexadeciltrimetil amônio (g)

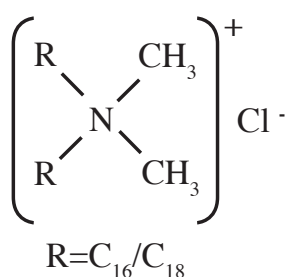

d)<smiles>[R][N+](C)(C)CC[CH-][CH2-]</smiles>

e)<smiles>[R][N+](C)(C)CCc1ccccc1</smiles>

$\mathrm{R}=\mathrm{C}_{12} / \mathrm{H}_{14}$

f)

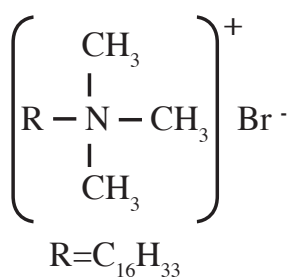

g)

Figura 4: Curvas TG e estruturas moleculares dos sais quaternários de amônio.

[Figure 4: TG curves and molecular structures of quaternary ammonium salts.]

cloreto de benzil se o íon cloreto atacar o grupo benzil [13]. Quase todos os sais de amônio investigados são líquidos iônicos, que devido à alta polaridade facilitam as reações. A partir de alguns resultados reportados [13], concluiu-se que os ânions cloretos poderiam ser removidos através de alguns processos de purificação ou lavagem durante a etapa de organofilização e com isso, o caminho de degradação via eliminação de Hoffmann poderia ser controlado.

A Tabela I apresenta os valores a $10 \%$ e $50 \%$ obtidos por TG para os sais quaternários de amônio. São apresentados valores de temperaturas de degradação $\left(\mathrm{T}_{10 \%}\right.$ e $\left.\mathrm{T}_{50 \%}\right)$, nas taxas de aquecimento de $10^{\circ} \mathrm{C} / \mathrm{min}$ e $12,5^{\circ} \mathrm{C} / \mathrm{min}$ e a fração de resíduo não volátil a $400{ }^{\circ} \mathrm{C}$. Por meio do estudo das curvas TG é possível investigar o comportamento térmico do material quando diferentes gases de purga são utilizados na realização dos ensaios. Observam-se variações na temperatura referente a $10 \%$ de degradação na taxa de 12,5 ${ }^{\circ} \mathrm{C} / \mathrm{min}$ de até $200{ }^{\circ} \mathrm{C}$. Já para as temperaturas referentes a $50 \%$ de degradação, diferenças de aproximadamente 106 ${ }^{\circ} \mathrm{C}$. Quando avaliados na taxa $10{ }^{\circ} \mathrm{C} / \mathrm{min}$ foram observadas variações similares de temperaturas. Verifica-se para todas as variações que o sal quaternário de amônio CET apresenta temperaturas de degradação superiores aos demais sais em estudo. Estes resultados justificam a explicação já comentada anteriormente que os sais à base do ânion cloreto se degradam em temperaturas inferiores enquanto o sal à base do ânion brometo se degrada em temperatura superior. No caso da utilização de atmosfera dinâmica de ar a degradação ocorreu a uma temperatura menor. Este comportamento já era esperado visto que o oxigênio acelera o processo de degradação térmica. É interessante observar que o percentual de resíduo a $400{ }^{\circ} \mathrm{C}$ do sal de amônio PRA 
Tabela I - Valores de temperaturas (T10\% e T50\%) e percentual de resíduo obtido por TG dos sais quaternários de amônio. [Table I - Values of temperatures (T10\% and T50\%) and residual percentage obtained by TG of the quaternary ammonium salts.]

\begin{tabular}{cccccccc}
\hline SAL & $\begin{array}{c}\mathrm{T} 10 \%\left({ }^{\circ} \mathrm{C}\right) \\
12,5^{\circ} \mathrm{C} / \\
\min \mathrm{Ar}\end{array}$ & $\begin{array}{c}\mathrm{T} 10 \%\left({ }^{\circ} \mathrm{C}\right) \\
12,5^{\circ} \mathrm{C} / \\
\min \mathrm{N}_{2}\end{array}$ & $\begin{array}{c}\mathrm{T} 50 \%\left({ }^{\circ} \mathrm{C}\right) \\
12,5{ }^{\circ} \mathrm{C} / \\
\operatorname{min~} \mathrm{Ar}\end{array}$ & $\begin{array}{c}\mathrm{T} 50 \%\left({ }^{\circ} \mathrm{C}\right) \\
12,5{ }^{\circ} \mathrm{C} / \\
\operatorname{min~} \mathrm{N}_{2}\end{array}$ & $\begin{array}{c}\mathrm{T} 10 \%\left({ }^{\circ} \mathrm{C}\right) \\
10{ }^{\circ} \mathrm{C} / \mathrm{min}\end{array}$ & $\begin{array}{c}\mathrm{T} 50 \%\left({ }_{2}{ }^{\circ} \mathrm{C}\right) \\
10{ }^{\circ} \mathrm{C} / \mathrm{min}\end{array}$ & $\begin{array}{c}\text { Resíduo em ar } \\
400{ }^{\circ} \mathrm{C}(\%)\end{array}$ \\
\hline PRA & 93 & 93 & 275 & 296 & 84 & 284 & $12,5{ }^{\circ} \mathrm{C} / \mathrm{min} \mathrm{Ar}$ \\
GEN & 68 & 78 & 260 & 266 & 61 & 262 & 0,23 \\
DOD & 51 & 53 & 190 & 190 & 49 & 185 & 1,35 \\
$\mathrm{CET}$ & 249 & 251 & 280 & 284 & 246 & 280 & 3,37 \\
\hline
\end{tabular}

é de $14 \%$, sendo este valor bastante superior aos outros sais, provavelmente devido ao elevado percentual de matéria ativa $(75 \%)$ na constituição do PRA e também devido à presença de uma mistura de cadeias carbônicas de $\mathrm{C}_{16} \mathrm{e}$ $\mathrm{C}_{18}$. Este valor está de acordo com as curvas TG onde se verificou que os demais sais de amônio possuem tamanhos de cadeias carbônicas de $\mathrm{C}_{16}$ sendo o PRA constituído de uma mistura de $\mathrm{C}_{16} \mathrm{e}_{18}$.

\section{CONCLUSÕES}

Os resultados de DSC e TG indicam que os sais à base do ânion cloreto começam a se degradar em temperaturas similares, enquanto o sal à base do ânion brometo se degradou em temperatura superior. Devido a uma estrutura química diferenciada, o maior comprimento de cadeia e a maior temperatura de decomposição, o sal PRA foi o escolhido para dar continuidade a estudos com a sua intercalação em argilas nacionais. Possivelmente, devido a essas características, esse sal apresentará uma boa interação com matrizes poliméricas.

\section{AGRADECIMENTOS}

Os autores agradecem à Clariant pela doação do sais, à RENAMI (Rede de Nanotecnologia Molecular e de Interfaces), ao MCT/CNPq e à CAPES/PROCAD-NF pelo apoio financeiro.

\section{REFERÊNCIAS}

[1] F. S. Poletto, A. R. Pohlmann, S. S. Guterres, Ciência Hoje 43, 255 (2008) 29.

[2] S. F. Wang, Y. Hu, L. Song, Z. Z. Wang, Z. Y. Chen, W.C. Fan, Polym. Degrad. Stab. 77 (2002) 423.

[3] M. Alexandre, P. Dubois, Mater. Sci. Eng. 28 (2000) 1.

[4] J. W. Gilman, Appl. Clay Sci. 15 (1999) 31.

[5] C. L. V. José, C. A. Díaz, P. M. Buchler, Sinterização de Argilas Organofílicas Visando seu Uso no Controle Ambiental de Resíduos de Fenol, Anais do $46^{\circ}$ Cong. Bras. Cerâmica, S. Paulo, SP (2002).

[6] A. B. Martins, Obtenção de argilas organofílicas purificadas para uso em fluidos de perfuração base óleo, Relatório ANP/PRH-25 (2007).

[7] J. W. Jordan, J. Phys. Chem. 53 (1949) 294.

[8] G. Lagaly, Clays Clay Minerals 30 (1982) 215.

[9] X. F. Qutubuddin, Polymer 42 (2001) 807.

[10] W. Xie, Z. Gao, W-P. Pan, D. Hunter, A. Singh, R. Vaia, Chem. Mater. 13 (2001) 2979.

[11] J. M. Cervantes-Uc, J. V. Cauich-Rodríguez, H. Vázquez-Torres, L. F. Garfias-Mesías, D. R. Paul, Thermochim. Acta 457 (2007) 92.

[12] R. D. Davis, J. W. Gilman, T. E. Sutto, J. H. Callahan, P. C. Trulove, H. C. De Long, Clays and Clay Minerals 52 (2004) 171.

[13] L. Cui, D. M. Khramov, C. W. Bielawski, D. L. Hunter, P. J. Yoon, D. R. Paul, Polymer 49 (2008) 3751.

(Rec. 20/11/2009, Ac. 27/03/2010) 